\title{
Electrodermal orienting to stimulus omission
}

\author{
J. G. O'GORMAN and JACQUELINE E. M. LLOYD \\ University of New England, Armidale, New South Wales, Australia
}

\begin{abstract}
The study examined whether complete omission of a tone stimulus following its repeated presentation would elicit an electrodermal orienting response and whether the factors of stimulus significance and subject lability influenced the probability and magnitude of any response to omission. A statistically significant increase in response on omission trials over that in control periods without stimulation preceding omission was observed. The omission effect was more likely to occur with labile subjects, those showing high levels of nonspecific electrodermal activity, than with stabiles; stimulus significance was not found to exert any systematic effect. Contrary to expectation, a measure of EEG alpha activity included in the study failed to show the omission effect. The failure was attributed to problems of measurement. Incidental findings of the study refute an arousal interpretation of electrodermal lability.
\end{abstract}

Of the various tests used by Sokolov to demonstrate that the orienting reflex (OR) is sensitive to stimulus novelty and not to any specific parameters of stimulation, the most compelling is that involving the omission of a stimulus. According to Sokolov (1969), if a stimulus is omitted from a series, an OR is elicited at the point in time the stimulus is scheduled to occur. An OR in these circumstances cannot be explained by reference to any physical features of the stimulus, since no stimulus is present, and must be seen as the result of stimulus change or novelty. Evidence for the omission effect is to be found in studies by Voronin and Sokolov (1960), Voronin, Bonfitto, and Vasilieva (1975), and O'Gorman and Lloyd (1976), and is most clearly expressed in the case of the EEG alpha blocking component of the OR. If components of the cortical evoked response are accepted as components of the OR, the omission effect is further supported by a number of studies of the P300 (e.g., Picton, Hillyard, \& Galambos, 1974; Ruchkin \& Sutton, 1973).

In contrast to studies of EEG components, studies of autonomic components of the OR, such as pupillary dilation (Cooper, Ashe, \& Weinberger, 1978) and electrodermal and vasomotor responses (Siddle \& Heron, 1975), have found little support for an omission effect. Siddle and Heron, in the most extensive investigation of the question to date, examined the influence of length of the stimulus series, interstimulus interval, and stimulus intensity. The only variable of systematic importance they could identify was the individual difference factor of fre-

This work has benefited from discussions with David Siddle, from the reading of an as yet unpublished manuscript by Robert Barry, and from the comments, on an earlier version of this article, of Edward Katkin and Keith Wilson and another (anonymous) reviewer. Requests for reprints should be sent to John O'Gorman, Department of Psychology, University of New England, Armidale, N.S.W., Australia 2351. quency of nonspecific electrodermal responses (NSRs). Subjects identified in post hoc analyses of the data as showing high frequencies of NSRs (labiles) were more likely to show an electrodermal OR to stimulus omission than were subjects showing low frequencies of NSRs (stabiles).

In discussing their results, Siddle and Heron suggested that either autonomic components of the OR were not as sensitive to omission of a stimulus as the EEG component or the factors of stimulus significance, subject set or expectancy, and length of training interfere in attempts to demonstrate the omission effect. There is some support in the data of Voronin et al. (1975) for the influence of stimulus significance. The omission of indifferent stimuli, that is, stimuli to which no particular response was required by the subject, gave rise to alpha blocking and skin potential responses only for stimuli presented at 12 to $15 \mathrm{sec}$. For stimuli presented at intervals of 2 to $3 \mathrm{sec}$ or $30 \mathrm{sec}$ or more, no omission effect was observed. However, for signal stimuli, those to which a motor response was required of the subject, an omission effect for EEG alpha and the skin potential response was observed for all interstimulus intervals.

The present study sought to examine the influence of stimulus significance and electrodermal lability on the skin conductance response to stimulus omission. The procedure followed was similar in many respects to that adopted in the study by O'Gorman and Lloyd which had successfully demonstrated an omission effect with EEG alpha. There were a total of 34 trials, with the stimulus being omitted on Trials 11 , 22 , and 33. Subjects were classified in advance as labiles and stabiles, and for one half of the subjects the significance of the stimulus was increased by the requirement to make a motor response to it.

One of the difficulties in research on the omission effect is defining what constitutes a response on the omission trial. The simplest definition (definition 1) 
is any response of criterion magnitude occurring within a latency window on the omission trial corresponding to the latency window used in defining a response to stimulus presentation (the standard latency window). This is, however, a rather lax definition in that it takes no account of the possible confounding of omission responses with spontaneous or nonspecific responses, the origin of which is independent of stimulus omission. This problem is particularly acute when lability is being studied as a correlate of the omission effect, because labiles, by definition, show more nonspecific responses and hence are more likely to be identified as showing the omission effect if any response within the standard latency window on the omission trial is taken as a sufficient condition for the effect.

There are two ways of dealing with the problem. The first is to define the omission effect as (definition 2) a response occurring within the standard latency window on the omission trial that is larger in magnitude than the response on the preomission trial in the habituation series. That is, an omission effect is demonstrated if, following habituation, omission leads to dishabituation of the OR. This definition may be too stringent, however, because response on the preomission trial is partly a function of the intensity of the stimulus and intensity is entirely lacking on the omission trial. A response may occur as a consequence of omission but not necessarily be of greater magnitude than the response on the preomission trial. A more reasonable point of comparison is the magnitude of nonspecific responding in a period prior to omission. The omission effect is then (definition 3) a response occurring within the standard latency window on the omission trial that is larger in magnitude than any response in a period without the habituation stimulus prior to the omission trial.

A further possible definition of the omission effect focuses not on the omission trial itself but on the trial that follows it. On the assumption that omission of the stimulus evokes an OR that interferes with the process of habituation, the omission effect could be defined (definition 4) as response on the postomission trial of larger magnitude than response on the preomission trial. This definition of the omission effect compares responses with events of equal intensity that occur within the same standard latency window. It does not, however, lead to an unequivocal demonstration of an omission effect, because increased response on the postomission trial could be attributed to spontaneous recovery, that is, reinstatement of response to the stimulus following a period without stimulus presentation (cf. Groves \& Thompson, 1970). Although spontaneous recovery is usually studied after longer periods (e.g., $3 \mathrm{~min}$ or more) without stimulation than obtains in omission studies to date (e.g., $60 \mathrm{sec}$ or less), it is nonetheless theoretically possible to appeal to spontaneous recovery in explaining any increased responding on the postomission trial.

Of the various definitions reviewed, definition 3 is the most defensible for demonstrating the omission effect with an autonomic component of the OR such as the electrodermal response. For this reason, it was used as the primary definition of the effect in the data analyses reported here. Analyses that followed from the other definitions were also performed, however.

\section{METHOD}

\section{Subjects and Design}

The design of the study was a $2 \times 2 \times 2 \times 3$ factorial with repeated measures on the last factor (trial blocks). The betweensubjects factors were sex, lability (labiles/stabiles), and stimulus condition (signal/nonsignal). Some analyses included trials as a further within-subjects factor.

Subjects were selected for the experiment in terms of their sex and their lability. The latter factor was assessed during a 5-min resting period prior to the experiment proper. Based on data on the distribution of lability (Siddle, personal communication), subjects were classified as labiles if they showed 11 or more NSRs in the 5min resting period and as stabiles if they showed 10 or fewer. Labiles and stabiles of each sex were allocated to the signal and nonsignal stimulus conditions in equal numbers until each condition had 24 subjects.

\section{Apparatus and Materials}

Skin conductance was recorded using $\mathrm{Ag}-\mathrm{AgCl}$ electrodes, $12 \mathrm{~mm}$ in diameter, constructed according to the specifications of Venables and Martin (1967) and attached with KY jelly, as the electrolyte, to the medial phalanges of the first and second finger of the subject's left hand. A constant voltage of $0.5 \mathrm{~V}$ was applied across the electrodes, using the system described in Venables and Christie (1973), and skin conductance was recorded on one channel of a Grass 7 polygraph with a 7P1B preamplifier sensitivity of $1 \mathrm{~mm}$ of pen deflection equal to $0.02 \mu \mathrm{mho}$.

EEG was recorded using $\mathrm{AgAgCl}$ electrodes, $10 \mathrm{~mm}$ in diameter, and commercial EEG paste, as the electrolyte, placed occipitally and contralaterally to the preferred hand $\left(C_{3}, O_{1}\right.$ or $C_{4}$, $\mathrm{O}_{2}$ ). The signal was amplified by a Grass $7 \mathrm{P} 3$ ac preamplifier and recorded on a second channel of the polygraph. Output from the $7 \mathrm{P} 3$ was also fed to a locally built integrator that was $3 \mathrm{~dB}$ down at 9 and $13 \mathrm{~Hz}$ and had a roll-off of $30 \mathrm{~dB}$. The integrator summed the amplitude of all activity within the 9 to $13 \mathrm{~Hz}$ band every 2 sec. Output from the integrator appeared as a spike on a third channel of the polygraph, and was measured in arbitrary units of millimeter deflection. Paper speed was $2.5 \mathrm{~mm} / \mathrm{sec}$.

The stimulus was a $1000-\mathrm{Hz}$ pure tone of $1-\mathrm{sec}$ duration produced by a Rapar audio-generator and presented to the subject through headphones. Intensity was $70 \mathrm{~dB}$, as measured at the headphones using a Dawe sound-level meter. The interstimulus interval was $20 \mathrm{sec}$ and was controlled by a Devices Digitimer, which also controlled stimulus duration. It was activated by the EEG integrator so that the 2-sec epochs for which EEG was integrated were measured from stimulus onset. The digitimer was also used to activate a Lafayette timer at the onset of the stimulus. The timer was stopped by depression of a buttonpress mounted in a plastic cylinder that fitted into the subject's hand.

Two questionnaires were administered to the subject. The first administered following the 5-min resting period, consisted of 10 semantic differential scales that sought the subject's reaction to the experimental environment. The descriptors used were: pleasantunpleasant, tense-relaxed, usual-unusual, clear-confusing, understandable-mysterious, predictable-unpredictable, simple-complex, useful-useless, and significant-trivial. A postexperimental ques- 
tionnaire was concerned with the subject's reactions during the experiment. It also included three questions from Kimble's (1967) scale of attitudes to psychological experiments.

\section{Procedure}

Subjects were recruited for the experiment on the understanding that it was concerned with the study of physiological changes induced by simple auditory stimuli. Following attachment of the electrodes, the subject was asked to relax as much as possible in a comfortable chair so that the equipment could be calibrated. If the subject was wearing a watch, this was removed before the electrodes were attached. Once the subject had been prepared for recording, a 5-min period elapsed during which frequency of NSRs was monitored. At the end of this period, the subject either continued with the experiment or was dismissed if he or she was classified on the basis of the lability score as belonging to a category for which a sufficient $\mathrm{n}$ had been obtained. Subjects continuing with the experiment completed the semantic differential questionnaire and were given instructions appropriate to the experimental condition to which they had been assigned.

For both conditions, the instructions began by indicating that a series of tones, which would be audible but not intense enough to be uncomfortable, would be heard from time to time through the headphones. For subjects in the nonsignal condition, the instructions then advised the subject that they were not required to do anything during the period of tone presentation but sit quietly and relax. Subjects in the signal condition were told that the experimenters were concerned with speed of reaction time, and that whenever they heard a tone they were to depress as rapidly as possible the key they had been given to hold and that their speeds would be recorded on an instrument in the adjoining room. The instructions concluded with a request to all subjects that they keep their eyes closed throughout the experiment. After positioning the headset, the experimenter retired to the adjoining room which housed the stimulus programming and recording apparatus. At the completion of the stimulus series, the experimenter again entered the subject's room and administered the second questionnaire.

\section{RESULTS}

Unless otherwise specified, the degrees of freedom for all $F$ tests reported in this section are 1,40. That is, a conservative test of significance, following the Greenhouse and Geisser procedure (Winer, 1962), was employed for all factors with repeated measures. Significant effects are those for which $\mathrm{p}<.05$.

\section{Skin Conductance}

A preliminary analysis compared groups in terms of skin conductance level (SCL) at five equidistant points during the resting period and during the three blocks of 10 tone trials separated by the omission trial. For each block, the SCL prior to each of the even-numbered trials was used in the analysis. There were no significant effects for lability $(F<1)$, sex, condition, trials, or blocks, and no significant interactions.

A skin conductance response (SCR) to the stimulus was taken to be any response of criterion magnitude $(\geqslant 0.02 \mu \mathrm{mho})$ occurring within $5 \mathrm{sec}$ of stimulus onset (the standard latency window). Magnitude of SCR to the tone was analyzed for the 10 trials in each of the three trial blocks. Analysis of variance indicated significant main effects for stimulus condition
$(F=13.84)$, trials $(F=10.27)$, and blocks $(F=8.64)$. The only significant interaction was that due to trials and blocks $(F=6.67)$. Magnitude of response was greater during the signal condition $(\mathrm{M}=0.66 \mu \mathrm{mho})$ than during the nonsignal condition ( $M=0.15 \mu \mathrm{mho})$ and was greater for earlier than for later trials during the first trial block. The effects for lability $(F=2.79)$ and sex $(F=1.49)$ were not significant, nor were the interactions between these and the other factors.

A similar analysis was performed on probability of response, that is, whether or not on any trial a response occurred. This yielded significant main effects for stimulus condition $(F=45.75)$, trials $(F=8.09)$, blocks $(F=25.16)$, and lability $(F=6.21)$. The only significant interaction was that between condition and blocks. The mean response probability fell from .91 in Block 1 to .83 in Block 2 to .78 in Block 3 for the signal condition, but the decrease in the nonsignal condition was from .57 to .28 to .25 . The least significant difference test indicated significant differences between all three blocks in the nonsignal condition. In the signal condition, the decrease from Block 2 to Block 3 was not significant. The main effect for trials was brought about by the decrease in response probability over trials. The main effect for lability was due to the higher response probability for labiles $(M=.69)$ than for stabiles $(M=.51)$.

The effects of stimulus omission were studied using the definitions outlined earlier. Definition 1 accepts any response of criterion magnitude occurring within the standard latency window on the omission trial. In all, 23 subjects responded in terms of this definition on one or more of the omission trials. This included 17 of the 24 labiles and 6 of the 24 stabiles, a difference significant by chi-square $\left[\chi^{2}(1, N=48)=\right.$ 10.10]. Thirteen were in the signal condition and 10 were in the nonsignal condition, a difference that is not significant $\left[\chi^{2}(1, N=48)=0.75\right]$.

Next, magnitude of response on the omission trials $(\mathrm{M}=0.06 \mu \mathrm{mho})$ was compared with that on the tone trials immediately preceding them $(M=0.37 \mu \mathrm{mho})$ (see definition 2 above). Only 7 of the 48 subjects showed a response on one or more of the omission trials larger in magnitude than that on the preceding trial. Five of these were labiles, and two were in the signal condition. Analysis of variance indicated main effects for condition $(F=15.23)$ and trials $(F=$ 15.45), and a significant interaction between condition and trials $(F=13.99)$. The effects for lability $(F=2.95)$, sex $(F=1.08)$, and blocks $(F=.76)$ were not significant, nor were any of the interactions including these factors. The significant interaction between condition and trials was not consistent with response to omission of the stimulus inasmuch as it was brought about by greater response on the preomission $(M=0.64 \mu \mathrm{mho})$ than on the omission trial $(M=$ $0.06 \mu \mathrm{mho}$ ) for subjects in the signal condition.

The third definition involves comparing response 
magnitude on the omission trial with response magnitude in an interval between the preomission and omission trials. The 10-15-sec interval following the preomission trial was used for this purpose. When this definition was applied, 18 of 24 labiles and 7 of the 24 stabiles were considered to have responded, a difference significant by chi-square $\left[\chi^{2}(1, N=48)=\right.$ 10.10]. A similar comparison in the case of stimulus significance indicated that 15 of the 24 subjects in the signal condition and 10 of the subjects in the nonsignal condition showed an omission effect, a difference that is not significant $\left[\chi^{2}(1, N=48)=2.08\right]$. Analysis of variance indicated significant main effects for lability $(F=4.28)$, trials $(F=8.28)$, and blocks $(F=5.19)$, but no significant interactions. The lability effect resulted from the fact that labiles $(M=$ $0.07 \mu \mathrm{mho}$ ) showed larger magnitude responses than stabiles $(\mathrm{M}=0.03 \mu \mathrm{mho})$. The trials effect was the consequence of the fact that response on the omission trial $(\mathbf{M}=0.06 \mu \mathrm{mho})$ was greater than response in the preomission period $(\mathrm{M}=0.02 \mu \mathrm{mho})$. The blocks effect resulted from a general decrease in responsiveness from Block $1(\mathrm{M}=0.09 \mu \mathrm{mho})$ to Block 3 $(\mathrm{M}=0.03 \mu \mathrm{mho})$.

Finally, omission was examined by comparing magnitude of response on the postomission trial with magnitude of response on the preomission trial (definition 4). In all, 20 labiles and 17 stabiles responded in terms of this definition on one or more of the test trials, 20 subjects under the RT condition and 17 under the no-RT condition. Analysis of variance showed a significant main effect for condition $(F=$ 10.40) and a significant interaction between trials (the pre-post comparison) and blocks $(F=3.62)$. The former effect was due to greater response magnitude in the signal $(\mathrm{M}=0.69 \mu \mathrm{mho})$ than in the nonsignal condition $(\mathrm{M}=0.15 \mu \mathrm{mho})$. The latter was brought about by greater response on the pre- than on the postomission trial for Block 1 only. For the first trial block, that is, responses surrounding the omission of the stimulus on Trial 11, magnitude of response on the preomission trial was larger $(M=0.37 \mu \mathrm{mho})$, although not significantly so, than response on the postomission trial $(\mathrm{M}=0.35 \mu \mathrm{mho})$. For the second trial block, response on the preomission $(\mathrm{M}=0.33 \mu \mathrm{mho})$ and postomission $(\mathbf{M}=0.51, \mu \mathrm{mho})$ trials were significantly different. This was also the case for Trial Block 3 (means of 0.40 and $0.46 \mu \mathrm{mho}$ ) for the preand postomission trials, respectively).

\footnotetext{
Alpha

Alpha activity during the rest period preceding the tone trials was measured for 4-sec periods every $30 \mathrm{sec}$. Analysis of variance on these data showed no significant effects for lability, sex, condition, or sampling period (all Fs, with the exception of that for sampling period, were less than unity). An analysis over trials for alpha activity in the 2 -sec period fol-
}

lowing stimulus presentation indicated a significant main effect for lability $(F=4.87)$. Labiles showed higher levels of alpha than did stabiles (means of 14.92 and $8.05 \mathrm{~mm}$, respectively). No other main effects or interactions reached significance.

In examining the omission effect, alpha activity on the omission trials was measured in the 2 -sec period following omission and on the pre- and postomission trials in the 2 -sec periods following stimulus presentation. The nonstimulus period used in applying definition 3 was the 14- to 16 -sec period following the preomission trial.

Similar results were obtained for all analyses directed to the three definitions of the omission effect. These indicated a significant main effect for lability, with labiles showing more alpha, but no other significant main effects or interactions. For the analysis in which omission and preomission trials were compared, the F value for lability was 5.45 . In the other two analyses, that comparing the omission trial and the nonstimulus period and that comparing the postomission and preomission trials, the $F$ values for lability were 5.65 and 4.78 , respectively. With only the lability effect significant, these analyses provide no evidence of response to stimulus omission for the EEG measure used here.

\section{Reaction Time and Self-Report Data}

For the 24 subjects in the signal condition, an analysis of reaction time over trials indicated main effects for lability $(F=6.47)$ and trials $(F=7.84)$. The lability effect was due to faster reaction times for stabiles $(M=217.6 \mathrm{msec})$ than for labiles $(M=$ $257.6 \mathrm{msec}$ ). The trials effect simply reflected the improvement in reaction time with practice.

The questionnaires administered to all subjects gave rise to scores on 14 scales. Only one of these showed a statistically significant discrimination among groups. Labiles described the experimental environment as more familiar than did stabiles $(F=$ 11.33), even though for all subjects this was their first experience in the laboratory. Two of the scales sought the subject's perception of degree of nervousness and tension, one being administered at the conclusion of the 5-min resting period and the other at the conclusion of the tone trials. Neither showed any difference between stabiles and labiles (Fs of 1.68 and 1.46 , respectively).

\section{DISCUSSION}

The purpose of the present study was to demonstrate, if possible, an omission effect with the electrodermal component of the OR and to examine the possible moderating role of lability and stimulus significance. If definition 3 above is accepted as including the necessary and sufficient conditions for demonstrating the omission effect described by 
Sokolov (1969), then the results of the present study support such an effect for the electrodermal component of the OR. Response magnitude in the standard latency window following stimulus omission was significantly larger than that in a period without stimulus presentation preceding it, and approximately half the sample showed an omission effect on at least one of the three omission trials. It is only if the omission effect is defined as a response on the omission trial greater than that to the stimulus on the preomission trial that the present results fail to show an omission effect. But, as argued earlier, such a definition is too stringent because it leaves out of consideration the influence of properties of the stimulus other than novelty in eliciting the response.

Analysis of the role of lability and stimulus significance in producing the effect pointed to the importance of the former but not the latter. Consistent with the observations of Siddle and Heron (1975), labile subjects were more likely to show an omission effect than were stabiles. A similar comparison for frequency of the omission effect between signal and nonsignal groups was not statistically significant. It should be noted, however, that lability was not significantly related to the magnitude of the omission response, because there was no significant interaction between lability and trials in the analysis of variance. Lability would thus seem to have more to do with whether or not an OR is elicited by stimulus omission than with its magnitude once elicited.

Stimulus significance was not found to exert a significant influence on either the probability or magnitude of the omission effect. It could be argued that the manipulation of stimulus significance in the present study confounded the effects of increased attention to the stimulus with increased motor activity. Because subjects in the signal condition were required to press to onset of the stimulus, any response in the interval following onset could be attributed to the effects of the motor act of pressing. It would have been more appropriate, in terms of this argument, to have had subjects press to stimulus offset and to have used a stimulus of duration sufficient to separate the effects of attention and motor activity. The argument loses some force, however, in the light of the experiments of Ray, Piroch, and Kimmel (1977), which compared the effects on electrodermal activity of pressing to stimulus onset and offset in a reaction time task. Their results indicated that motor activity per se was not responsible for the increased electrodermal OR when pressing to stimulus onset. Of greater relevance, however, is the consideration that any confounding would only have occurred when the omission effect was defined in terms of the comparison of response on the omission and preomission trials. For the omission effect defined in terms of definition 3, the primary definition adopted here, no confound is involved. Buttonpressing did not ac- company the omission trial or the comparison interval prior to omission, and hence could not exert an influence on the omission effect defined in this way. The conclusion that stimulus significance, as manipulated here, is not important in demonstrating the omission effect is consistent with the results of O'Gorman and Lloyd (1976). In that study, buttonpressing to stimulus offset was found not to exert a statistically reliable influence on the EEG alpha response to omission. Elsewhere (O'Gorman, 1979), it has been argued that stimulus significance is of less importance for elicitation of the OR than is claimed by some theorists.

The other factor examined in the analysis of variance for its influence on the omission effect was repetition (the trial blocks factor). That the omission effect was not sensitive to repetition may be considered something of a difficulty for interpretation of the effect observed in terms of OR theory, inasmuch as an OR should habituate with repeated presentation of the events that elicit it. That is, the OR to omission on Trial 33 should have been less than that to omission on Trial 11. This was not the case, although there was, consistent with OR theory, a general decrease in responsiveness from Trial 11 to Trial 33. However, only three repetition trials were presented, and these were spaced at intervals of over 3 min. Study of repetition on the omission effect needs to be explored systematically in further work, using shorter intervals and more trials.

The most troubling feature of the present data was the failure to demonstrate an omission effect with the measure of EEG alpha activity, since previous work had suggested that the effect was most reliably demonstrated with EEG components. The failure to demonstrate it here can be attributed to a number of factors, the first and most important being that the EEG component of the OR was not adequately measured. The analysis of alpha activity over training trials showed no significant effect for trials, that is, there was no reliable evidence of habituation for the EEG measure. As habituation is a defining property of the OR, the relevance to Sokolov's theory of results with this measure must be questioned. Failure to show habituation may in turn have been due to the time period selected for integration. Two seconds may have been too long to show alpha blocking to the stimulus with sufficient sensitivity. In our previous study, alpha blocking was measured by hand from the EEG record. An integrator was introduced in the present study to increase the objectivity of the measurement process, but the end result may have been to hide the essential effect. Reanalysis of the EEG data was not possible because the paper speed employed did not permit a manual analysis, and data were not collected on magnetic tape.

A further departure from the procedures employed in the previous study, as far as the EEG was con- 
cerned, was the failure to select subjects in terms of their resting level of spontaneous alpha. In that study, only "good" alpha subjects were selected and the selection criteria employed were strict, as evidenced by the rejection ratio of $35 \%$. In the present study, subjects were not selected in terms of resting alpha; in fact, explicit selection in terms of another variable, nonspecific electrodermal activity, was dictated by the purposes of the study. The extent to which demonstration of the omission effect with EEG alpha depends on selection of good alpha subjects must await further research.

Although the major aim of the study was demonstration of an omission effect, the results provide some useful information about the lability variable. Labiles and stabiles did not differ in terms of resting alpha levels, SCL, or self-reported levels of nervousness and tension during the experiment. They did differ, however, in terms of SCR probability, in EEG alpha abundance, and in reaction time to tone stimulation. Interestingly, it was the stabiles who showed the faster reaction times and the lower levels of alpha, but the labiles who showed less decline (habituation) in probability of SCR. These data are not consistent with an interpretation of lability differences based on arousal theory, a common, though disputed, account of such differences (Katkin, 1975). Faster reaction times and lowered alpha activity would be considered in terms of that theory as the concomitants of increased arousal and hence should accompany high and not low frequencies of NSRs. Although of less force, the lack of difference between labiles and stabiles in terms of SCL and resting alpha activity is also inconsistent with an arousal interpretation of lability.

Although contrary to expectations based in arousal theory, the findings are not without precedent in the literature. The pattern of intercorrelation of magnitude, base level, and NSR frequency is similar to that reported by Martin and Rust (1976) in their factor-analytic investigation of the structure of electrodermal measures, whereas the finding that labiles and not stabiles have slower reaction time is consistent with the results of Kleinman and Stern (1968), although it must be noted that there are other studies that have shown the reverse effect, notably those by Lacey and Lacey (1958). In interpreting their results, Kleinman and Stern reasoned that conditions that facilitate ORs should, through the inhibition of motor activity, a concomitant of the OR according to Sokolov (1963), lead to slowing of RTs. In the present study, labiles maintained ORs to the imperative stimulus and showed slower RTs. An interpretation of lability differences in terms of attentional rather than arousal processes would thus seem more satisfactory.

\section{REFERENCES}

Cooper, C. L., Ashe, J. H., \& Weinberger, N. M. (1978). Effects of stimulus omission during habituation of the pupillary dilation reflex. Physiological Psychology, 6, 1-6.

Groves, P. M., \& Thompson, R. F. (1970). Habituation: A dual process theory. Psychological Review, 77, 419-450.

KATK IN, E. S. (1975). Electrodermal lability: A psychophysiological analysis of individual difference in response to stress. In I. G. Sarason \& C. D. Spielberger (Eds.), Stress and anxiety (Vol. 2). New York: Wiley.

Kimble, G. A. (1967). Attitudinal factors in eyelid conditioning. In G. A. Kimble (Ed.), Foundations of conditioning and learning. New York: Appleton-Century-Crofts.

Kleinman, K. M., \& Stern, J. A. (1968). Task complexity, electrodermal activity and reaction time. Psychophysiology, 5 , $51-60$.

LACEY, J. I., \& LACEY, B. C. (1958). The relationship of resting autonomic activity to motor impulsivity. Research Publication of the Association for Nervous and Mental Diseases, 36, 144-209.

Martin, I., \& RUST, J. (1976). Habituation and the structure of the electrodermal system. Psychophysiology, 13, 554-562.

O'Gorman, J. G. (1979). The oriention reflex: Novelty or significance detector? Psychophysiology, 16, 253-262.

O'Gorman, J. G., \& Lloyd, J. E. M. (1976). Alpha blocking to the omission of a stimulus. Physiological Psychology, 4, 285-288.

Picton, T. W., Hillyard, S. A., \& Galambos, R. (1974). Cortical evoked responses to omitted stimuli. In M. N. Livanov (Ed.), Major problems in brain electrophysiology (pp. 302-311). Moscow: U.S.S.R. Academy of Sciences.

Ray, R. L., Piroch, J. F., \& Kimmel, H. D. (1977). The effect of task and stimulus variability on habituation of electrodermal and vasomotor reactions. Physiological Psychology, 5, 189-196.

Ruchkin, D. S., \& Sutton, S. (1973). Visual evoked and emitted potentials and stimulus significance. Bulletin of the Psychonomic Society, 2, 144-146.

Siddle, D. A. T., \& Heron, P. A. (1975). Stimulus omission and recovery of the electrodermal and digital vasoconstrictive components of the orienting response. Biological Psychology, 3, 277-293.

Sokolov, E. N. (1963). Perception and the conditioned reflex. Oxford: Pergamon.

Sokolov, E. N. (1969). The modelling properties of the nervous system. In M. Cole \& I. Maltzman (Eds.), A handbook of contemporary Soviet psychology. New York: Basic Books.

Venables, P. H., \& Christie, M. J. (1973). Mechanisms, instrumentation, recording techniques, and quantification of responses. In W. F. Prokasy \& D. C. Raskin (Eds.), Electrodermal activity in psychological research. New York: Academic Press.

Venables, P. H., \& Martin, I. (1967). Skin resistance and skin potential. In P. H. Venables \& I. Martin (Eds.), A manual of psychophysiological methods. Amsterdam: North Holland.

Voronin, L. G., Bonfitto, M., \& Vasilieva, V. M. (1975). The interrelation of the orienting reaction and conditioned reflex to time. In E. N. Sokolov \& O. S. Vinogradova (Eds.), Neuronal mechanisms of the orienting reflex. Hillsdale, $\mathrm{NJ}$ : Erlbaum.

Voronin, L. G., \& Sokolov, E. N. (1960). Cortical mechanisms of the orienting reflex and its relation to the conditioned reflex. Electroencephalography and Clinical Neurophysiology, Suppl. 13, 335-346.

WINER, B. J. (1962). Statistical principles in experimental design. New York: McGraw-Hill.

(Manuscript received February 20, 1984; revision accepted for publication May $18,1984$. 